\title{
Measurements of the direction of the solar wind using interplanetary scintillation
}

\author{
P. J. Moran ${ }^{1}$, A. R. Breen ${ }^{1 *}$, C. A. Varley $^{1}$, P. J. S. Williams ${ }^{1}$, W. P. Wilkinson ${ }^{1}$, J. Markkanen ${ }^{2}$ \\ ${ }^{1}$ Physics Department, University of Wales Aberystwyth, Penglais, Aberystwyth SY23 3BZ, UK \\ ${ }^{2}$ EISCAT Sodankylä, SF-99600, Finland
}

Received: 10 October 1997 / Revised: 27 March 1998 / Accepted: 3 April 1998

\begin{abstract}
EISCAT observations of the interplanetary scintillation of a single source were made over an extended period of time, during which the orientation of the baselines between the two observing sites changed significantly. Assuming that maximum correlation between the scintillations observed at the two sites occurs when the projected baseline is parallel to the direction of plasma flow, this technique can be used to make a unique determination of the direction of the solar wind. In the past it has usually been assumed that the plasma flow is radial, but measurements of eleven sources using this technique have indicated conclusively that in at least six cases observed at mid or high heliocentric latitude there is a significant non-radial component directed in four cases towards the heliocentric equator and in two cases towards the pole.
\end{abstract}

Key words. Solar physics - Astrophysics · Astronomy · Magnetic fields · Space plasma physics $\cdot$ Charged particle motion and acceleration

\section{Introduction}

For many years observations of interplanetary scintillation (IPS) - the fluctuation in radio power received from a compact radio source when the line of sight passes close to the Sun - have been used to calculate the velocity of the solar wind (e.g. Dennison and Hewish, 1967). The cross-correlation function between the scintillations recorded by two spaced antennas can be compared with a theoretical function which models

Correspondence to: P.J. Moran

* Present address: Max-Planck-Institut für Aeronomie, D-37191 Katlenburg-Lindau, Germany weak scattering along the line of sight by irregularities which are carried outward by the solar wind (Armstrong and Coles, 1972). Such measurements can be made at all heliocentric latitudes and over a wide range of distances from the Sun, from inside 10 solar radii to beyond the Earth's orbit. However, as the method requires weak scattering, in order to cover the whole range of solar distances it is necessary to use a corresponding range of observing frequencies, with measurements closer to the Sun requiring higher frequencies.

The correlation between the scintillations in the signals received by two antennas is highest when the baseline between the two antennas lies in the same plane as the flow of the solar wind. On the assumption that this flow is close to radial, the optimum time for measuring solar wind velocity occurs when the ray paths from the source to the two antennas lie in the same radial plane, and because the orientation of the baseline varies as the Earth rotates (see Fig. 1) EISCAT observations are normally scheduled for only $15 \mathrm{~min}$ centred at this optimum time. However, from 1994 onwards a number of these observations have been extended for periods of up to $2 \mathrm{~h}$ in order to check whether maximum correlation did indeed occur when the baseline was parallel to the radial plane (Breen et al., 1996a, b)

Some off-radial flow in the solar wind has been predicted theoretically in association with transient events and corotating interaction regions (CIRs) (Pizzo, 1978, 1980, 1982), and in situ data taken by the Helios, Voyager and Pioneer spacecraft have been analysed to investigate both azimuthal and meridional flow (Alexander and De La Torre, 1995; Pizzo et al., 1983; Marsh and Richter, 1984; Bala and Prabhakaran Nayar, 1993; Richardson and Paularena, 1996). This work, however, was mainly concerned with off-radial components in the flow of the solar wind close to the ecliptic plane. Multiantenna IPS has also been used in an attempt to measure solar wind direction close to the Sun (e.g. Armstrong et al., 1986).

The purpose of the EISCAT IPS experiment was to measure the flow direction with the highest attainable 


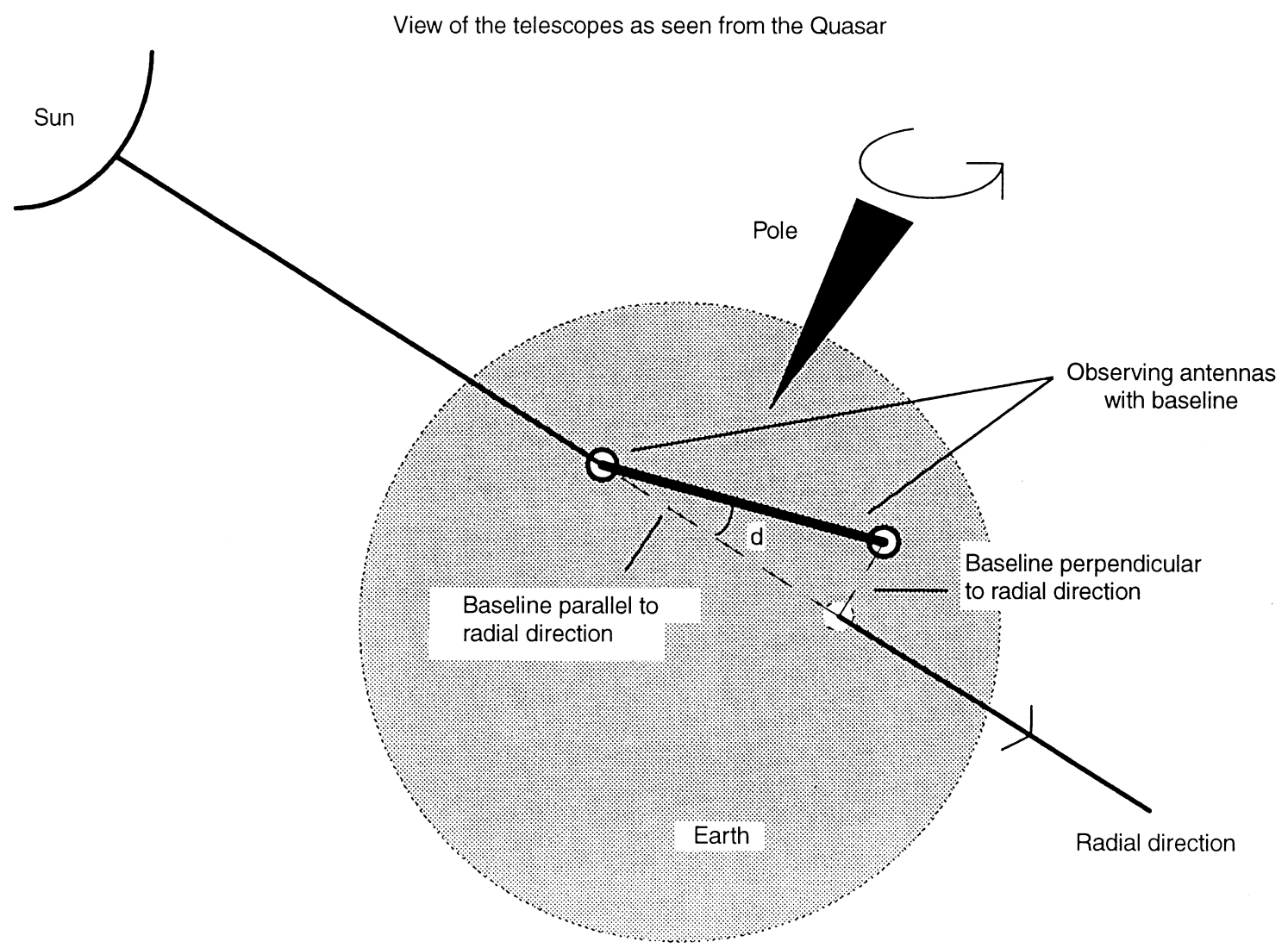

Fig. 1. The orientation of the baseline between the two antennas in relation to the radial plane through the centre of the Sun, which varies as the Earth rotates about its axis

accuracy at mid or high latitudes for solar distances between 30 and 100 solar radii (Breen et al., 1996a, b; Moran et al., 1996). In reporting the initial results from the EISCAT IPS experiment Breen et al. (1996a, b) had suggested the possibility of measuring off-radial flow and presented some preliminary examples. The present paper gives the results of a careful analysis of eleven extended observations which show that at least in some cases there is a systematic meridional component in the flow of the solar wind, usually directed from the pole to the equator.

\section{Observations and analysis}

Figure 2 shows how the maximum of the cross-correlation function varies with the projected baseline perpendicular to the radial direction for one of the extended observations. Each point corresponds to $10 \mathrm{~min}$ of observation. A small correction is made to the perpendicular baseline to allow for the movement of the Earth around the Sun during the time-lag at which maximum cross-correlation occurs (in the case of EISCAT typically 0.2 to $0.5 \mathrm{~s}$ ). This adjustment reduces or increases the value of the perpendicular baseline for each data point, and depends on the heliocentric latitude and solar distance of the observation. In the eleven cases detailed in this paper, the correction ranges from 2.2 to $13.6 \mathrm{~km}$.
To allow for changes in the parallel baseline at the same time as the changes in the perpendicular baseline, the correlation values were also corrected for the variation of peak correlation with parallel baseline. To make this correction, it is necessary to know how the maximum cross-correlation varies with parallel baseline

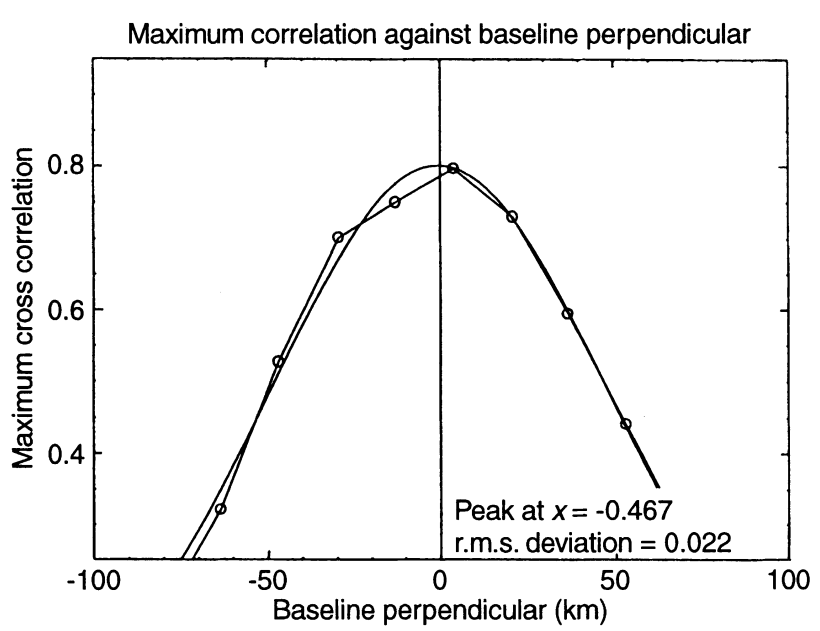

Fig. 2. The maximum correlation coefficient for different values of $b_{\perp}$, the perpendicular baseline. Each point represents the analysis of 10 min observation. The Gaussian curve fitted to these points has a peak value for $b_{\perp} \sim 0.5 \mathrm{~km}$ and the standard error in each point $\sigma_{10}=0.022$ (after allowing for the loss of 3 degrees of freedom in fitting) 
when the perpendicular baseline is fixed. Data were taken from the whole set of normal 15-min observations, selecting cases where the perpendicular baseline was close to zero, where the observations were of very high quality, and where fast streams accounted for at least $85 \%$ of the total scintillation. After applying these selection criteria, 31 data points were obtained. Figure 3 plots these values, showing how the maximum correlation observed $C$ varied with the parallel baseline $b_{\|}$. Various curves were fitted to these data and a $\chi^{2}$ test was applied in each case. The results showed that the most appropriate simple curve to represent the data was a Gaussian of the form:

$C\left(b_{\|}\right)=a \exp \left\{-b_{\|}^{2} / 2 \sigma_{\|}^{2}\right\}$.

If $\ln (C)$ is plotted against $b_{\|}^{2}$ and a weighted regression used to fit the best straight line to the data, the intercept of the line is an indication of $\ln (a)$ and the slope of the line an indication of $2 \sigma_{\|}^{2}$, giving values of $a=0.91 \pm 0.03$ and $\sigma_{\|}=285 \pm 30 \mathrm{~km}$. (This work will be discussed in more detail in a later paper dealing with the scale-size of the IPS diffraction pattern).

After correcting the initial data for the movement of the Earth and for changes in $b_{\|}$, the final values of maximum correlation coefficient were plotted as a function of $b_{\perp}$ and a least-squares method was used to fit a Gaussian curve to the points, as shown in Fig. 2. A curve that included skew and kurtosis as extra parameters was also fitted, and as measured by the 'raw' rms deviation this curve was a slightly better fit in each case although the improvement was always small. (For example the coefficient for the skew term was never greater than $3 \times 10^{-7}$, suggesting that skewness could be ignored). When allowance was made for the loss of one or two more degrees of freedom, it was clear that a simple Gaussian was the most appropriate fitted curve.

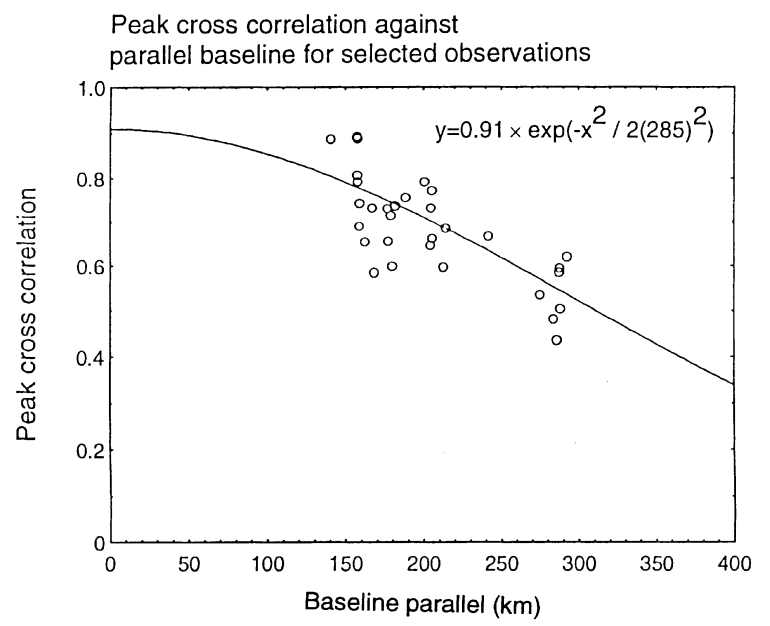

Fig. 3. The fall in maximum correlation coefficient as the parallel baseline increases. In this figure only high-latitude observations in which the fast-stream was $>85 \%$ dominant are shown. A Gaussian curve is fitted which gave the best least-squares fit out of a straight line, and exponential decay curve and a Gaussian curve
Figure 2 shows that the peak of the best-fitting Gaussian curve corresponded to a perpendicular baseline slightly offset from zero, nominally indicating that the flow of the solar wind was off-radial. However, with such a small offset from zero, it is necessary to consider whether this offset is significant or whether it is the combined result of random errors in the different measurements involved.

There are two sources of error to be considered:

1. uncertainty in the correction for changes in the parallel baseline during the $2 \mathrm{~h}$ of observation;

2. random noise errors in the original data.

The uncertainty in the correction for parallel baseline reflects the uncertainty in the parameters of the fitted Gaussian curve shown in Fig. 3. Of course this error only applies to the adjustment for changes in $b_{\|}$which, for the eleven observations reported in this study, is never more than $16 \%$ of the raw value. The dependency of this error on the deviation in parallel baseline means that the errors are greatest away from the centre of an observation. For a plot similar to Fig. 2, during which $b_{\|}$varies significantly, the total error in individual values of $C\left(b_{\perp}\right)$ as a result of this correction can be as large as 0.02 for the largest (or smallest) values of $b_{\|}$.

The effect of this uncertainty in $\sigma_{\|}$does depend on whether it introduces a random variance in each individual measurement, which should then be added to the variance due to noise, or whether it introduces a systematic error into the whole set of observations. If for example $b_{\|}$is decreasing throughout the observations then in the first half of the observations the measured value of maximum correlation must be increased and in the second half decreased to allow for the change. However, if $\sigma_{\|}$has been overestimated, then the corrected values will be too low on one side of the plot and too high on the other and there will be a systematic error in the measured offset. Such a systematic error has a more serious effect on the final result than the equivalent random errors, so in making a conservative estimate of the significance of any measured offset it is assumed that the error is systematic, and the variance in the measured offset due to this is added to the variance due to the noise errors.

In some cases, however, the correction for changes in the parallel baseline is very small, and in such cases the noise errors in the observations themselves prove to be the limiting factor. To determine these random errors a careful study was made of two extended observations where the parallel baseline remained almost constant for the whole of the run. An estimate of the noise error in the correlation coefficient determined from each $10 \mathrm{~min}$ of observation can be derived from the rms deviation of the nine observed points $y_{o, i}$ from the fitted Gaussian curve $y_{f, i}$, allowing for the three degrees of freedom spent in fitting the mean value, the amplitude and the width of the Gaussian curve

$\sigma_{10}=\sqrt{\frac{\sum_{1}^{9}\left(y_{o, i}-y_{f, i}\right)^{2}}{6}}$ 
In this case the calculation gives $\sigma_{10}=0.022$.

To check this value, the same raw data was reanalysed, but on this occasion each correlation function was based on only 5 min of data. A new Gaussian curve was fitted, as shown in Fig. 4, and the random noise error derived from the rms deviation of 18 points from the fitted Gaussian, once again allowing for the three degrees of freedom spent in fitting.

$\sigma_{5}=\sqrt{\frac{\sum_{1}^{18}\left(y_{o, i}-y_{f, i}\right)^{2}}{15}}$

In this case $\sigma_{5}=0.029$. With only half the information being used for each point it is expected that the spread about the fitted curve would be greater, and if the errors are truly random, the rms deviation from each point on the curve corresponding to $5 \mathrm{~min}$ of observation should be approximately $\sqrt{ } 2$ greater than the deviation of the points derived after $10 \mathrm{~min}$ of observation, i.e. $\sigma_{5} \approx 1.4 \sigma_{10}$. In fact $\sigma_{5}=1.3 \sigma_{10}$. This (and other similar results) is considered to be adequate confirmation that the deviation of each point from the best-fitting Gaussian curve is indeed due to random noise, and $\sigma_{10}$ is assumed to be the random error in each point in Fig. 2.

To determine the overall effect of these noise errors on the measured offset a Monte Carlo method was used to add simulated random noise errors, with an rms value of $\sigma_{10}$, to nine points $y_{f, i}$ spaced equally along the fitted Gaussian curve, thus generating a set of simulated points with the same level of noise errors as the real data. The least-squares method is used to fit a new Gaussian curve to the simulated data and so determine a new value for the offset. This procedure is repeated 1000 times and the different values of the offset obtained are used to determine the likely error in the measured offset and hence the significance level of the result.

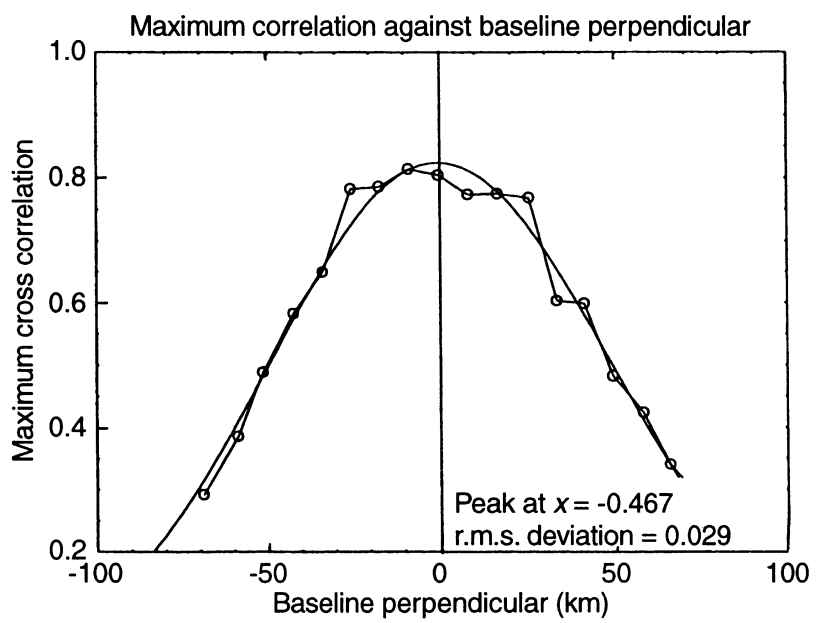

Fig. 4. The maximum correlation coefficient for different values of the perpendicular baseline. Each point represents the analysis of $5 \mathrm{~min}$ observation. The Gaussian curve fitted to these points has a peak value for $b_{\perp} \sim 0.5 \mathrm{~km}$ (as in Fig. 2) but in this case the standard error in each point $\sigma_{5}=0.029$ (the ratio $\sigma_{5}: \sigma_{10}$ is very close to $\sqrt{ } 2$ so there is no evidence that the errors are other than random)

\section{Results}

Each Monte Carlo analysis provides the probability distribution of the true offset in the perpendicular baseline for maximum correlation, which is assumed to correspond to the direction of solar-wind flow. It is then possible to calculate statistically the likelihood that the observed peak in the fitted curve is offset from zero by chance. Figure 5 shows two examples where the data indicate an off-radial component of velocity and the results are significant at $1 \%$ and $5 \%$, respectively. In other words, the chance that the apparent off-radial component was caused by random noise errors is less than $1 \%$ and $5 \%$.

So far the data from eleven extended observations have been analysed, and the results are detailed in Table 1. Nominally, eight of these show an Off-radial component directed towards the equator and three a component directed towards the pole. However, when
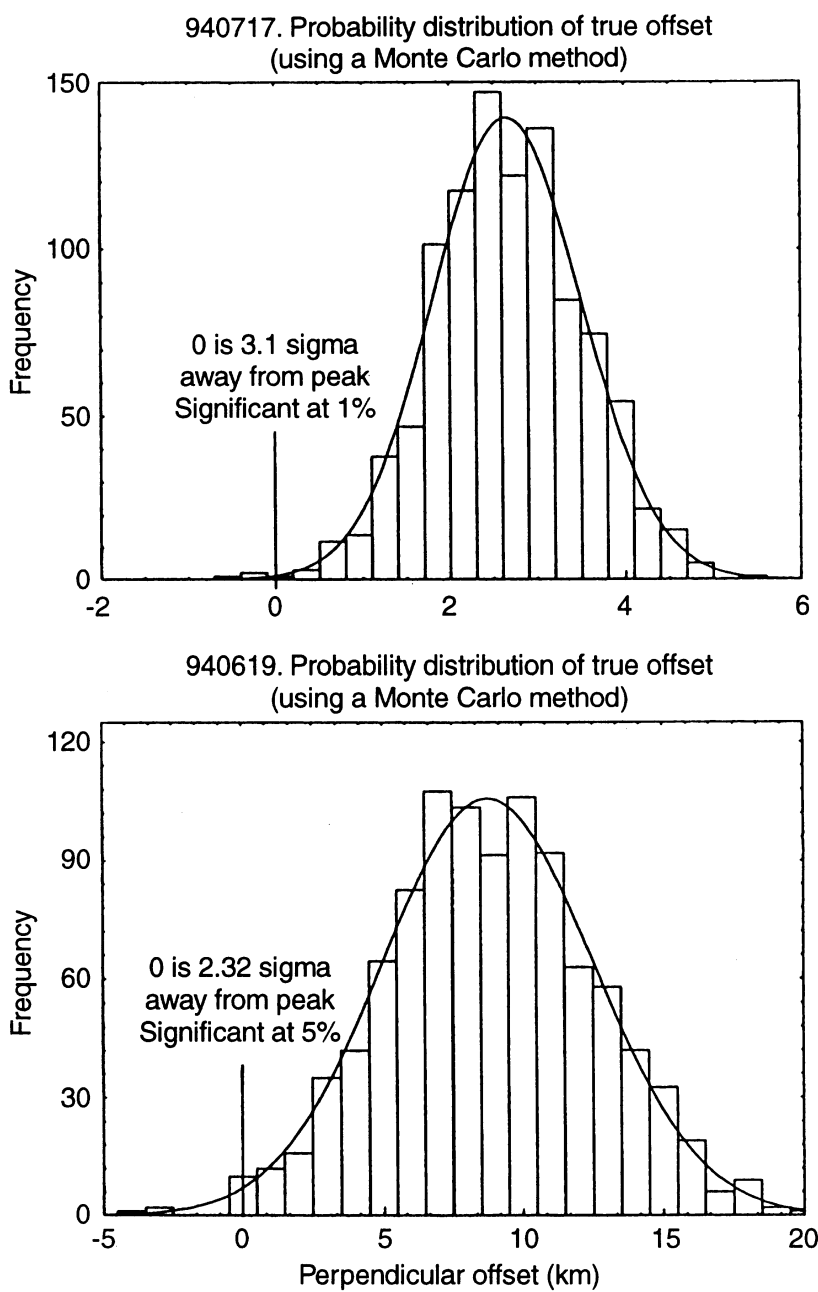

Fig. 5. Two examples where a Gaussian curve is fitted to the histogram of uncertainty generated by the Monte Carlo method. In the first case the parallel baseline was constant throughout the observations so there was no baseline correction. In the second case the uncertainty was greater due to the uncertainty in baseline correction. The offset is significant as $1 \%$ and $5 \%$ respectively. 
Table 1. Results from the eleven extended-run observations.

\begin{tabular}{|c|c|c|c|c|c|}
\hline $\begin{array}{l}\text { Date and } \\
\text { source }\end{array}$ & $\begin{array}{l}\text { Helio-centric } \\
\text { latitude }\left({ }^{\circ} \mathrm{N}\right)\end{array}$ & $\begin{array}{l}\text { Distance } \\
\text { (solar radii) }\end{array}$ & Offset angle $\left(^{\circ}\right)$ & $\begin{array}{l}\text { Statistical } \\
\text { significance }\end{array}$ & $\begin{array}{l}\text { Direction } \\
\text { (towards) }\end{array}$ \\
\hline $\begin{array}{l}94-06-05 \\
0521 \mathrm{p} 166\end{array}$ & -53.7 & 33.1 & $0.6 \pm 0.5$ & $20 \%$ & equator \\
\hline $\begin{array}{l}94-06-19 \\
0625 \mathrm{p} 146\end{array}$ & -51.2 & 44.7 & $1.6 \pm 0.7$ & $5 \%$ & equator \\
\hline $\begin{array}{l}94-07-17 \\
0745 \mathrm{p} 101\end{array}$ & -73.9 & 41.1 & $0.7 \pm 0.3$ & $1 \%$ & equator \\
\hline $\begin{array}{l}96-05-30 \\
0319 p 415\end{array}$ & 59.7 & 89.6 & $1.1 \pm 0.3$ & $0.1 \%$ & equator \\
\hline $\begin{array}{l}96-06-08 \\
0521 \mathrm{p} 166\end{array}$ & -72.7 & 26.4 & $-1.0 \pm 0.2$ & $0.1 \%$ & pole \\
\hline $\begin{array}{l}960622 \\
0521 \mathrm{p} 166\end{array}$ & -24.2 & 45.6 & $-0.8 \pm 0.5$ & $10 \%$ & equator \\
\hline $\begin{array}{l}96-06-24 \\
0625 \mathrm{p} 146\end{array}$ & -72.8 & 34.6 & $0.1 \pm 0.3$ & - & (equator) \\
\hline $\begin{array}{l}96-06-27 \\
0625 \mathrm{p} 146\end{array}$ & -77.9 & 32.3 & $0.0 \pm 0.3$ & - & \\
\hline $\begin{array}{l}96-08-17 \\
1008 \text { p143 }\end{array}$ & -30.8 & 28.3 & $0.6 \pm 0.5$ & - & (equator) \\
\hline $\begin{array}{l}96-09-16 \\
1120 \mathrm{p} 143\end{array}$ & 47.3 & 46.6 & $0.3 \pm 0.6$ & - & (pole) \\
\hline $\begin{array}{l}960922 \\
1118 \mathrm{p} 125\end{array}$ & 27.6 & 58.8 & $-2.5 \pm 0.8$ & $1 \%$ & pole \\
\hline
\end{tabular}

the significance of each measured offset is considered, only six cases are significant at $10 \%$ or less, of which four are significant at $1 \%$ or less. In four of these six cases, the apparent meridional flow is directed from the pole towards the equator, but in two cases the apparent offset is directed towards the pole.

\section{Discussion}

This method measures the apparent direction of solarwind velocity in a plane perpendicular to the line of sight. This direction can then be resolved into two components, one in the radial direction and the other close to the meridional direction, but care must be taken before interpreting these components in terms of the true radial and meridional components. Scattering occurs along the whole line of sight and although the scattering potential per unit volume falls off with solar distance as $R^{-4}$, so that the most important contribution comes from that sector of the ray path closest to the Sun, the analysis cannot ignore scattering at greater solar distances. The contribution of scattering from all points along the line of sight to the apparent radial component of velocity will be reduced by a ' $\cos \theta$ ' factor, known as the foreshortening effect. At the same time, the apparent meridional component may also contain a small contribution from any azimuthal velocity, where meridional and azimuthal components are defined using spherical co-ordinates in a heliocentric system.

However, geometry dictates that the contribution of any azimuthal component of the solar-wind velocity to the observed off-radial flow will be very small. Thus calculation shows that for a heliocentric latitude of 30 degrees, the fraction of any azimuthal velocity contributing to the apparent meridional component will range from $2 \%$ at 20 solar radii to $0.6 \%$ at 80 solar radii. In contrast, at the same solar distances the fraction of any true meridional flow contributing to the apparent meridional component will lie between $98 \%$ and $97 \%$. A similar pattern is calculated for the contribution of azimuthal velocity when the scintillating source is observed at a latitude of 60 degrees, ranging from $2 \%$ at 20 solar radii to $0.5 \%$ at 80 solar radii. In this case the proportion of the meridional velocity observed at the same distances ranges from $89 \%$ to $86 \%$.

\section{Conclusion}

A method has been developed to make accurate measurements of any non-radial components of the solar-wind flow in the meridional plane. For sources with a strong scintillating component, the noise errors are relatively small and if such sources are observed in a configuration where $b_{\|}$is almost constant throughout the period of observation, while $b_{\perp}$ changes from strongly positive to strongly negative, it is possible to measure the off-radial component with sufficient accuracy to demonstrate conclusively whether there is a meridional component of solar wind velocity directed from the pole towards the equator or vice versa.

It is well known that in the azimuthal plane, as a result of solar rotation, the plasma moves radially outwards while the magnetic field has a spiral configuration, but in the meridional plane this does not apply and the direction of the magnetic field is parallel to the direction of plasma flow. As the off-radial velocity measured by this method is almost entirely in the meridional plane it follows that the results presented indicate the direction of the large-scale magnetic field of the Sun, averaged over the whole line of sight. For four of the six cases where the measurement is significant at $10 \%$ or less the off-radial velocity is directed from the 
pole towards the equator, suggesting a dipolar magnetic field, but in the other cases the offset is towards the pole.

This is the first time that directional measurements of this accuracy have been made at high heliocentric latitude and at distances between 30 and 100 solar radii. Non-radial flow is expected near the fast-stream/slowstream interface of a co-rotating interaction regions (CIRs), the development of which becomes important at distances beyond $1 \mathrm{AU}$, and in the vicinity of coronal mass ejections (CMEs). However in all the cases reported in the present paper only a fast stream is observed along the whole line of sight and there is no evidence of any CIRs or CMEs. It follows that for the solar distances and latitudes at which these observations are made it is likely that the non-meridional flow can be associated with magnetic and pressure imbalances between high and low latitudes.

At present, the two limiting factors in these measurements are the uncertainty in the correction for parallel baseline and the high level of system noise, especially for observations made at Tromsø. In future the effect of both factors can be reduced significantly.

To reduce the uncertainty in the correction for $b_{\|}$two avenues will be followed. First, care will be taken to identify all cases where a source can be observed by two antennas for $2 \mathrm{~h}$ with almost constant $b_{\|}$, or at least with $b_{\|}$varying symmetrically about the time when $b_{\perp}$ is zero. In addition more observations will be used to study the way that maximum correlation falls as $b_{\|}$increases, as shown in Fig. 3. In this way it will be possible to identify how this relationship varies with sunspot cycle, solar distance, heliocentric latitude, and between fast and slow streams in the solar wind and as a result to derive a more reliable correction algorithm.

The noise errors in the present observations largely correspond to the high system noise of the receiving system at Tromsø. Because of the need to protect the receiver at Tromsø from the transmitted signal when the antenna is being used as part of an active radar, the system noise at this station will always be higher than at Kiruna and Sodankylä. Thus between 1994 and 1997 the system noise temperature at Tromsø for observations near the zenith was about $105 \mathrm{~K}$ while at the other two stations it was about $35 \mathrm{~K}$. However, with the introduction of cooled GaAsFET preamplifiers and an improved 'receiver-protect' component and the removal of a redundant amplitude-hybrid from the antenna feed, the system noise temperature at Tromsø could be reduced to $70 \mathrm{~K}$ or even less, resulting in an equivalent reduction in the noise errors. Funds have now been provided by PPARC in the UK and MPI für Aeronomie in Germany to make this improvement.

With a marked improvement in the error-level at Tromsø it will become worthwhile to improve the system noise level at Kiruna and Sodankylä. At present only one polarisation channel is available at each of these stations and re-activation of the orthogonal channel will double the information flow - and reduce the rms noise level by a factor of 0.71 - in each case.
Once all these possibilities are realised this method will be used regularly to 'map' the direction of the solar wind flow and the associated magnetic field at solar distances and heliocentric latitudes which are normally inaccessible in any other way. The interpretation of the results in terms of the pressure balance and magnetic field direction throughout the heliosphere will be the subject of a later paper.

Acknowledgements. The authors wish to thank the Director and Staff of EISCAT for their help in this work, and Professor W. A. Coles of the Department of Electrical and Computer Engineering, University of California for his collaboration that enabled this research project to begin. Three of us (PJM, CAV and RAF are funded by PPARC).

The Editor-in-chief thanks E. A. Lucek and another referee for their help in evaluating this paper.

\section{References}

Alexander, P., and A. De La Torre, The solar wind angular momentum and energy carried by the interplanetary magnetic field, Solar Phys. 157, 367-373, 1995.

Armstrong, J. W., and W. A. Coles, Analysis of three-station interplanetary scintillation, J. Geophys. Res., 77, 4602-4610, 1972.

Armstrong, J. W., W. A. Coles, M. Kojima, and B. Rickett, The sun and heliosphere in three dimensions Doraschet Reidel, 1986.

Bala, B., and S. R. Prabhakaran Nayar, IPS measurements and azimuthal variation of solar-wind velocity of the heliospheric current sheet, Planet. Space Sci., 41, 127-131, 1993.

Breen, A. R., W. A. Coles, R. Grall, U-P Løvhaug, J. Markkanen, H. Misawa, and P. J. S. Williams, EISCAT measurements of interplanetary scintillation, J. Atmos. Terr. Phys., 58, 507-519, 1996a.

Breen, A. R., W. A. Coles, R. Grall, M. T. Klinglesmith, J. Markkanen, P. J. Moran, B. Tegid, and P. J. S. Williams, EISCAT measurements of the solar wind, Ann. Geophysicae, 14, 1235-1245, 1996b.

Dennison, P. A., and A. Hewish, The solar wind outside the plane of the ecliptic, Nature, 213, 343-346, 1967.

Marsh, E., and A. K. Richter, Distribution of solar wind angular momentum between particles and magnetic field: inferences about the Alfvén critical point from Helios observations, $J$. Geophys. Res., 89, 5386-5394, 1984.

Moran, P. J., A. R. Breen, W. A. Coles, R. Grall, M. T. Klinglesmith, J. Markkanen, C. A. Varley, and P. J. S. Williams, EISCAT measurements of the solar wind: measurements of fast and slow streams, Phys. Chem. Earth Solar Sys., 22, 389-392, 1996.

Pizzo, V., A three-dimensional model of corotating streams in the solar wind 1. Theoretical foundations, J. Geophys. Res., 83, 5563-5572, 1978.

Pizzo, V., A three-dimensional model of corotating streams in the solar wind 2. Hydrodynamic streams, J. Geophys. Res., 85, 727743, 1980.

Pizzo, V., A three-dimensional model of corotating streams in the solar wind 3. Magnetohydrodynamic streams, J. Geophys. Res., 87, 4374-4394, 1982.

Pizzo, V., R. Schwenn, E. Marsh, H. Rosenbauer, K.-H. Mühlhäuser, and F. M. Neubauer, Determination of the solar wind angular momentum flux from the HELIOS data - an observational test of the Weber and Davis theory, Astrophys. J., 271, 335-354, 1983.

Richardson, J. D., and K. I. Paulerena, Meridional flow in the solar wind, J. Geophys. Res., 101, 19995-20002, 1996. 